\title{
P4HA1 regulates human colorectal cancer cells through HIF1 $\alpha$-mediated Wnt signaling
}

\author{
QIANG ZHANG ${ }^{1}$, YUE YIN $^{2}$, HONGYE ZHAO $^{3}$, YAN SHI $^{4}$, WEI ZHANG ${ }^{5}$, \\ ZHENGPENG YANG ${ }^{1}$, TINGTING LIU ${ }^{1}$, YONGHONG HUANG ${ }^{6}$ and ZHANJIANG YU ${ }^{1}$
}

\begin{abstract}
Departments of ${ }^{1}$ General Surgery and ${ }^{2}$ Scientific Research, The Third Affiliated Hospital of Qiqihar Medical University, Qiqihar, Heilongjiang 161000; Departments of ${ }^{3}$ Physiology and ${ }^{4}$ Biochemistry, Qiqihar Medical University, Qiqihar, Heilongjiang 161006; ${ }^{5}$ Department of Endocrinology, The Third Affiliated Hospital of Qiqihar Medical University, Qiqihar, Heilongjiang 161000; ${ }^{6}$ Department of Biochemistry, Qiqihar Medical University, Qiqihar, Heilongjiang 161006, P.R. China
\end{abstract}

Received June 12, 2020; Accepted September 25, 2020

DOI: $10.3892 / \mathrm{ol} .2020 .12406$

\begin{abstract}
Colorectal cancer (CRC) is the third most commonly diagnosed malignancy that is associated with high levels of mortality. CRCs are often associated with an aberrant wingless-type mouse mammary tumor virus integration site family (Wnt) signaling pathway known to be responsible for tumorigenesis and cancer progression. Other factors that contribute to CRC pathology include hypoxia, extracellular matrix and cellular microenvironment. In the present study, modulation of Wnt, a common molecular progenitor for CRC-associated pathology was evaluated. CRC tissues and specific cell lines were found to exhibit increased expression levels of prolyl 4-hydroxylase subunit $\alpha 1$ (P4HA1). P4HA1 expression was found to stabilize hypoxia inducible factor- $1 \alpha(\mathrm{HIF} 1 \alpha)$. The silencing of P4HA1 resulted in decreased cell proliferation, cell cycle arrest in the $\mathrm{G}_{1}$ phase, decreased tumorsphere formation, decreased tumorsphere volume, increased susceptibility to 5-fluorouracil and increased caspase-3 activity. However, P4HA1 silencing resulted in the activation and thus proteasomal degradation of $\beta$-catenin, indicative of the abrogation of Wnt signaling pathway. Wnt is a critical signaling pathway and is activated in most CRCs. HIFl $\alpha$ is a poor prognostic marker in CRC. The present study provided preliminary evidence that HIF1 $\alpha$ and the Wnt signaling pathway in CRC are modulated through P4HA1. P4HA1 may serve not just as a biomarker for CRC prognosis but may also be targeted for potential therapeutic intervention.
\end{abstract}

Correspondence to: Dr Zhanjiang Yu, Department of General Surgery, The Third Affiliated Hospital of Qiqihar Medical University, 5 Taishun Street, Qiqihar, Heilongjiang 161000, P.R. China

E-mail: 33163864@qq.com

Key words: prolyl 4-hydroxylase subunit $\alpha 1$, colorectal cancer, hypoxia inducible factor- $1 \alpha$, Wnt signaling

\section{Introduction}

Previously, colorectal cancer (CRC) was characterized by an aging population in high income countries attributed to dietary habits and lifestyle (1). However, currently CRC is the third most commonly diagnosed malignancy and ranks fourth in terms of mortality with 2900,000 deaths annually, including in developing countries $(2,3)$. While $\mathrm{CRC}$ rates vary widely worldwide, three distinct temporal patterns have been identified based on incidence and mortality rates. The pattern of increased incidence and increased mortality is most often seen in transitioning economies (4). One such economy that suffers from the burden of cancer and associated mortality is China. The incidence of CRC (12.2\%) was found to be second only after lung cancer (18.1\%) (3). The burden of the disease not only equates to the associated mortality but also severely impacts the quality of life.

Improving the mortality rate of CRC can be approached by improving diagnostic techniques and providing early interventional treatment. However, CRC may in part be derived from a variety of environmental factors, making it one of the most heterogenous types of cancer (5). Its complex heterogeneity is not yet completely understood and contributes to the lack of predictive prognostic markers, except for the $R A S$-mutation status (6).

Genomics and epigenomics have revolutionized cancer diagnostics and predictive biomarker assessments (7); therefore, the present study aimed to elucidate the influence of post-translation modifications (PTM) on carcinogenesis in CRC. The era of -omics (genomics, transcriptomics and epigenomics) coupled with technological advancements, such as next generation sequencing, has greatly improved the understanding of different cancers (8). However, translation of these in silico findings to the field is largely affected by the lack of available data.

One of the most common PTM is prolyl hydroxylation. Hydroxyproline constitutes $\sim 4 \%$ of all amino acids, with most of them being present in collagen. In human pancreatic cancer cells and tissues, prolyl 4-hydroxylase subunit $\alpha 1$ (P4HA1), is the most predominant isoform that contributes to proline 
4-hydroxylase activity (9). Proline 4-hydroxylase comprises tetrameric units $(\alpha 2 \beta 2)$ and is responsible for the 4-hydroxylation of proline, ensuring folding and formation of the collagen triple helix (10). The role of P4HA1 has been investigated in breast cancer (11) and pancreatic cancer (9). Whilst the epigenetic modification of P4HA1 with microRNAs (miR) have received attention in prostate cancer (12), the contribution of prolyl hydroxylation in CRC tumorigenesis remains to be elucidated. It has previously been shown in both triple negative breast cancer cells and pancreatic ductal adenocarcinoma that P4HA1 stabilizes hypoxia inducible factor-1 $\alpha$ (HIF1 $\alpha)$ through modulating glycolytic activity, such as the levels of $\alpha$-ketoglutarate and succinate, thus mediating cellular transformation (10).

It has been demonstrated that the wingless-type mouse mammary tumor virus integration site family (Wnt) signaling pathway is activated in CRCs $(13,14)$. The Wnt proteins are secreted glycoproteins that also act as ligands of the Wnt signaling pathway. There are three distinct Wnt signaling pathways currently known: i) $\beta$-catenin pathway; ii) the calcium dependent pathway; and iii) planar cell polarity pathway (14). While cell growth and proliferation are promoted through the canonical $\beta$-catenin pathway, cell motility and polarity are governed by the latter two pathways. Surface binding of intracytoplasmic $\beta$-catenin and Wnt ligands activates the Wnt signaling pathway (14). However, $\beta$-catenin can be phosphorylated in the absence of Wnt ligands (15).

Given the aforementioned evidence, the present study aimed to establishing the role of the most common PTM, $\mathrm{P} 4 \mathrm{HA} 1$, in the promotion and progression of CRC through the activation of the $\beta$-catenin pathway. In addition, the pathway through which P4HA1 alters the cell signaling pathways leading to tumor progression was assessed. With this objective, in silico analyses using the Gene Expression Omnibus (GEO) database confirmed the upregulation of P4HA1 in CRC (15). In vitro experiments analyzed the mRNA and protein expression of P4HA1 and its influence on cellular transformation, with respect to cellular proliferation, stemness and chemoresistance. This is a preliminary demonstration of the activation of the $\beta$-catenin pathway through P4HA1.

The assessment of PTMs and their signaling mechanisms may serve as a unique approach to expand the repertoire of reliable diagnostic and prognostic markers in CRC. Furthermore, these mechanisms may also serve as molecular targets for directed intervention.

\section{Materials and methods}

GEO analysis. We analyzed gene expression of CRC patients through two GEO databases, GEO/GDS4382 (https://www. ncbi.nlm.nih.gov/geo/query/acc.cgi?acc=GSE32323) (16) and GEO/GDS5232 (https://www.ncbi.nlm.nih.gov/geo/query/acc. cgi?acc=GSE25071) (17) using GEO2R method (https://www. ncbi.nlm.nih.gov/geo/info/geo2r.html).

Clinical samples. The present study was performed in accordance with the Declaration of Helsinki and approved by the Medical Ethics Committee of Qiqihar Medical University (no. 2015QY137). Written informed consent was obtained from all patients. Cancer tissues and adjacent normal tissues ( $\geq 5 \mathrm{~cm}$ from the edge of the tumor) were collected by debulking surgery from 30 patients with colorectal adenocarcinoma who were recruited into a clinical trial at the Third Affiliated Hospital of Qiqihar Medical University between January 2016 and January 2017. Clinicopathological information was obtained and two pathologists independently determined diagnoses according to World Health Organization classification of tumors of the digestive system (18) and the clinicopathological parameters listed in Table SI. None of the patients in the study received chemotherapy or radiation treatment prior to surgery and all patients were diagnosed with adenocarcinoma and other pathological types were excluded. Of the 30 included patients, 18 were men and 12 were women. The age of patients ranged from 42 to 81 years. The cancers were classified as follows: 8 well-differentiated, 15 moderately differentiated and 7 poorly differentiated colorectal tissues.

Human CRC cell culture. The human CRC cell lines (SW480, SW620 and HCT116 cells) were purchased from The Cell Bank of Type Culture Collection of the Chinese Academy of Sciences and cultured in Dulbecco's modified Eagle's medium (DMEM) supplemented with 10\% FBS (Gibco; Thermo Fisher Scientific, Inc.), $100 \mathrm{U} / \mathrm{ml}$ penicillin and $100 \mu \mathrm{g} / \mathrm{ml}$ streptomycin (Gibco; Thermo Fisher Scientific, Inc.). All cells were cultured at $37^{\circ} \mathrm{C}$ in a $5 \% \mathrm{CO}_{2}$ atmosphere. The cells were cultured in $1 \%$ oxygen atmosphere when hypoxic conditions were required. P4HA1 and control siRNA were purchased from Shanghai GenePharma Co., Ltd. These siRNA duplexes $(100 \mathrm{nmol} / \mathrm{l})$ were transfected into CRC cells using Lipofectamine ${ }^{\circledR} 2000$ (Invitrogen; Thermo Fisher Scientific, Inc.) according to the manufacturer's protocols. Briefly, one day before transfection, CRC cells were incubated in growth medium without antibiotics and were $\sim 50 \%$ confluent at the time of transfection. Lipofectamine 2000 was mixed with Opti-MEM I Reduced Serum Medium (Invitrogen; Thermo Fisher Scientific, Inc.) and incubated for $5 \mathrm{~min}$ at room temperature, then the siRNA duplexes were mixed with the diluted Lipofectamine 2000 and incubated for $20 \mathrm{~min}$ at room temperature. Subsequent experiments were performed in CRC cells $48 \mathrm{~h}$ post-transfection at $37^{\circ} \mathrm{C}$ in a $\mathrm{CO}_{2}$ incubator. The following sequences of siRNA were used for transfection: P4HA1 siRNA, 5'-GAUAAAGUCUCUGUUCUAG-3'; HIF1 $\alpha$, 5'-AACCAAGTAGCCTGTTATCAA-3'; and control siRNA, 5'-UUCUCCGAACGUGUCACGUTT-3'.

$R N A$ extraction and reverse transcription-quantitative $P C R$ $(R T-q P C R)$. All commercial assays used were performed according to the manufacturer's instructions. Total mRNA from cultured SW620 and HCT116 cells and tissue samples was extracted using TRIzol ${ }^{\circledR}$ reagent (Invitrogen; Thermo Fisher Scientific, Inc.),. Complementary DNA was synthesized from $2 \mu \mathrm{g}$ total RNA using a Reverse Transcription kit (Takara Biotechnology Co., Ltd.) according to the manufacturers' instructions. Real-time quantitative PCR analyses were performed with SYBR-Green Real-Time Master Mix (Applied Biosystems; Thermo Fisher Scientific, Inc.) on a 7500 Fast Real-Time PCR system (Applied Biosystems; Thermo Fisher Scientific, Inc.) using the following thermocycling conditions: 
Initial denaturation at $95^{\circ} \mathrm{C}$, followed by 30 cycles at $95^{\circ} \mathrm{C}$ for $15 \mathrm{sec}$ and $60^{\circ} \mathrm{C}$ for $1 \mathrm{~min}$. The following primer pairs were used for qPCR: P4HA1 forward, 5'-AGGGGTTGC TGTGGATTACC-3' and reverse, 5'-GTCATGTACTGTAGC TCGGC-3'; and GAPDH forward, 5'-GGGCTGCTTTTA ACTCTGGT-3' and reverse, 5'-TGGCAGGTTTTTCTAGAC GG-3'. The Applied Biosystems 7500 Fast software version 1.4 (Applied Biosystems; Thermo Fisher Scientific, Inc.) was used to analyze the $\mathrm{Cq}$ values of different. Comparisons were made using the $2^{-\Delta \Delta C t}$ method (19) and mRNAs normalized to an endogenous control GAPDH.

Immunohistochemical staining. CRC tissues were fixed in $10 \%$ formalin for one week at room temperature and embedded in paraffin, and then cut into 5- $\mu$ m-thick sections. The sections were deparaffinized in a xylene bath, rehydrated in PBS and then subjected to heat-induced epitope retrieval. The sections were then blocked with $3 \%$ hydrogen peroxide for $5 \mathrm{~min}$ at room temperature, incubated with primary antibodies against P4HA1 (cat. no. ab127564; 1:100; Abcam) at $4^{\circ} \mathrm{C}$ overnight. The sections were then washed with PBS, followed by incubation with HRP-conjugated secondary antibody (cat. no. sc-2357 mouse anti-rabbit IgG-HRP; 1:1,000; Santa Cruz Biotechnology, Inc.) for $1 \mathrm{~h}$ at room temperature. The sections were then visualized using a DAB (Sigma-Aldrich; Merck KGaA) according to the manufacturer's instructions under a light microscope (Nikon Corporation; magnification, 200x).

Western blotting. Total proteins were extracted from normal colorectal tissues, CRC tissues and SW480, SW620 and HCT116 cell lines using ice-cold RIPA lysis buffer [50 mM Tris (pH 7.4), 150 mM NaCl, 1\% Triton X-100, 1\% sodium deoxycholate, $0.1 \% \mathrm{SDS}$ ] with proteinase/phosphatase inhibitors (Thermo Fisher Scientific, Inc.). The protein concentration was measured by bicinchoninic acid method. Lysates containing $20 \mu \mathrm{g}$ protein were separated via SDS-PAGE (10\% gel), which were then transferred to polyvinylidene difluoride membranes (EMD Millipore). The membranes were blocked in 5\% non-fat milk (Thermo Fisher Scientific, Inc.) for $1 \mathrm{~h}$ at room temperature and subsequently incubated with primary antibodies against P4HA1 (cat. no. ab127564; 1:1,000; Abcam), $\beta$-catenin (cat. no. sc-7963; 1:1,000; Santa Cruz Biotechnology, Inc.), phosphorylated (p)- $\beta$-catenin (cat. no. sc-101650; 1:2,000; Santa Cruz Biotechnology, Inc.), CD133 (cat. no. sc-30220; 1:1,000; Santa Cruz Biotechnology, Inc.), Nanog (cat. no. sc-134218; 1:1,000; Santa Cruz Biotechnology, Inc.), axis inhibition protein 2 (Axin2; cat. no. sc-25302; 1:1,000; Santa Cruz Biotechnology, Inc.), c-Myc (cat. no. sc-373712; 1:1,000; Santa Cruz Biotechnology, Inc.) and GAPDH (cat. no. sc-20357; 1:500; Santa Cruz Biotechnology, Inc.). All antibodies were obtained from Santa Cruz Biotechnology, Inc. The membranes were then washed 3 times in $0.1 \%$ Tween ${ }^{\circledR} 20$ detergent and incubated with horseradish peroxidase-conjugated mouse anti-rabbit or anti-goat secondary antibodies (cat. nos. sc-2357 mouse anti-rabbit IgG-HRP and sc-2005 goat anti-mouse IgG-HRP; 1:10,000; Santa Cruz Biotechnology, Inc.) for $1 \mathrm{~h}$ at room temperature. Protein bands were detected using an enhanced chemiluminescence kit (Thermo Fisher Scientific, Inc.). ImageJ software (version 1.48; National Institutes of
Health, NIH) was used to determine intensity of western blot bands normalized to GAPDH.

MTS assay. The proliferation of SW620 and HCT116 cells was measured using an MTS assay kit (Promega Corporation) according to the manufacturer's instructions. Briefly, SW620 and HCT116 cells (2,000 cells/well) were seeded into 96-well plates and, after $24 \mathrm{~h}$, the medium was replaced with fresh DMEM and the cells were further cultured for 3 days at $37^{\circ} \mathrm{C}$. MTS reagent $(20 \mu \mathrm{l})$ was then added to each well, and plates were incubated for $1 \mathrm{~h}$ at $37^{\circ} \mathrm{C}$. Absorbance was measured at $490 \mathrm{~nm}$ using a microplate reader (Bio-Rad Laboratories, Inc.). Each individual experiment was performed with six replicates three independent times.

Cell Counting Kit-8 (CCK-8) assay. CCK-8 cell proliferation kit (Beyotime Institute of Biotechnology) was used to analyze CRC cell proliferation according to the manufacturer's protocol. SW620 and HCT116 cells $\left(0.5 \times 10^{4} /\right.$ well) were seeded into 96 -well plates and cultured overnight at $37^{\circ} \mathrm{C}$. The supernatant was then removed and $2 \mathrm{ml} \mathrm{CCK}-8$ reagent, WST-8, was added into each well and incubated for $1 \mathrm{~h}$ to be reduced by dehydrogenase in mitochondria to orange formazan. The absorbance was then measured at $490 \mathrm{~nm}$ using a microplate reader (Bio-Rad Laboratories, Inc.).

Cell cycle. SW620 and HCT116 cells $\left(2 \times 10^{6} / \mathrm{ml}\right)$ were collected and washed twice with PBS buffer and centrifuged at $1,000 \mathrm{xg}$ for $5 \mathrm{~min}$ at $4^{\circ} \mathrm{C}$. The supernatant was discarded and cells were fixed with ice-cold $70 \%$ ethanol (by adding dropwise to the pellet while vortexing) for at least $30 \mathrm{~min}$ at $4^{\circ} \mathrm{C}$. The cell solution was centrifuged at $1,000 \mathrm{x}$ for $5 \mathrm{~min}$ at $4^{\circ} \mathrm{C}$ and cells were washed twice with PBS and centrifuged again at 1,000 x g for $5 \mathrm{~min}$ at $4^{\circ} \mathrm{C}$. The supernatant was discarded and cells were incubated with $50 \mu \mathrm{l}$ RNase I ( $1 \mu \mathrm{g} / \mathrm{ml}$; Sigma-Aldrich; Merck $\mathrm{KGaA})$ at $37^{\circ} \mathrm{C}$ for $1 \mathrm{~h}$ in the dark. Cells were then treated with $200 \mu \mathrm{l}$ propidium iodide $(20 \mu \mathrm{g} / \mathrm{ml})$ at room temperature for $1 \mathrm{~min}$ according to the manufacturer's protocol. Cell cycle analysis was performed using the LSR II flow cytometer (BD Biosciences) and the data were analyzed by FlowJo software (version 9; FlowJo, LLC).

Caspase-3 activity assay. Caspase-3 activity was performed using the caspase-3 Activity Assay kit (Beyotime Institute of Biotechnology) following the manufacturer's protocol. In brief, proteins were isolated from CRC cells and $100 \mu \mathrm{g}$ protein were added to a reaction buffer containing $2 \mathrm{mM}$ peptide substrate acetyl-Asp-Glu-Val- Asp p-nitroanilide (Ac-DEVD-pNA) solution from the kit, and incubated at $37^{\circ} \mathrm{C}$ for $2 \mathrm{~h}$. The absorbance was measured using a microplate reader (Bio-Rad Laboratories, Inc.) at $405 \mathrm{~nm}$. The caspase-3 activity was recorded as the ratio to that of the control group.

Tumorsphere assay. SW620 and HCT116 cells were cultured in DMEM with $10 \% \mathrm{FBS}$ in an incubator containing $5 \% \mathrm{CO}_{2}$ at $37^{\circ} \mathrm{C}$. When cells reached $80 \%$ confluence, $6 \times 10^{5}$ cells were resuspended in stem cell culture medium supplemented with 2\% B27 (Gibco; Thermo Fisher Scientific, Inc.), 20 ng/ml recombinant human epidermal growth factor (Gibco; Thermo Fisher Scientific, Inc.) and $20 \mathrm{ng} / \mathrm{ml}$ recombinant human 
A

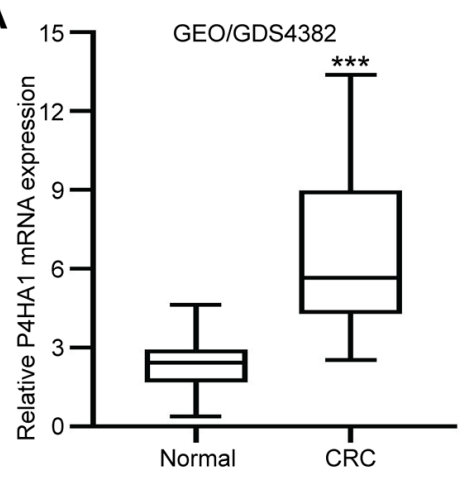

D

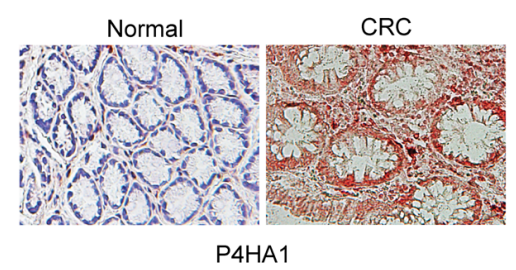

B

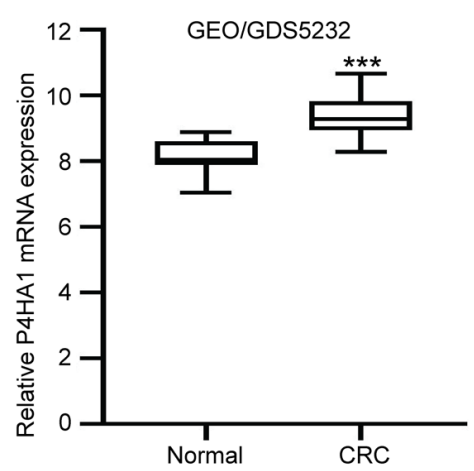

E
C

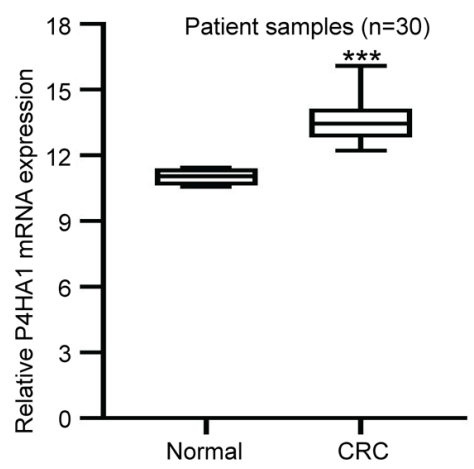

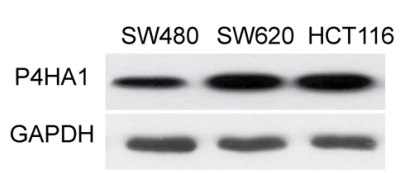

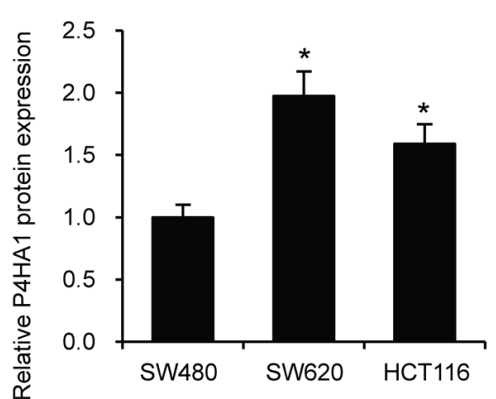

Figure 1. Upregulation of P4HA1 in CRC tissues and cells. (A and B) P4HA1 mRNA expression in CRC tissues and normal colorectal tissues from GEO database in (A) GEO/GDS4382 dataset and (B) GEO/GDS5232 dataset. P<0.001. (C) Expression of P4HA1 mRNA was examined in clinical CRC tissues and normal colorectal tissues. P $<0.001$. (D) Immunostaining of P4HA1 in clinical CRC tissue and normal colorectal tissue (magnification, $x 200$ ). (E) P4HA1 protein expression was examined in SW480, SW620 and HCT116 cells by western blot analysis. P4HA1 protein expression was quantified in the right panel. ${ }^{*} \mathrm{P}<0.05$ vs. SW480; ${ }^{* * *} \mathrm{P}<0.001$ vs. CRC. CRC, colorectal cancer; GEO, Gene Expression Omnibus; P4HA1, prolyl 4-hydroxylase subunit $\alpha 1$.

fibroblast growth factor (Gibco; Thermo Fisher Scientific, Inc.) in each 6-well ultralow adhesion plates (Corning Inc.) for 6 days at $37^{\circ} \mathrm{C}$ in a $5 \% \mathrm{CO}_{2}$ atmosphere. When the tumorspheres grew to $50 \mu \mathrm{m}$ in diameter, the spheres were imaged and counted under a light microscope (Nikon Corporation). The primary spheres were dissociated into single cells and cultured in stem cell culture medium (Gibco; Thermo Fisher Scientific, Inc.) for 14 days at $37^{\circ} \mathrm{C}$ to allow each cell forming one tumorsphere.

Chemoresistant assay. SW620 and HCT116 cells were treated with the chemotherapeutic agent 5-fluorouracil (5-FU) at various concentration $(0,2,4,8$ or $16 \mathrm{mmol} / \mathrm{l})$ for $2 \mathrm{~h}$, and the cells were then cultured for $24 \mathrm{~h}$. Subsequent experiments were performed.

Statistical analysis. Each experiment was performed three times. All results were analyzed using SPSS software, version 19.0 (IBM Corp.). Values are expressed as the mean \pm SD. Differences between two groups were analyzed using unpaired Student's t-test or one-way ANOVA followed by Bonferroni post hoc analysis for multiple group comparisons, as appropriate. $\mathrm{P}<0.05$ was considered to indicate a statistically significant difference.

\section{Results}

P4HAl is upregulated in CRC tissues and cells. The expression of P4HA1 was analyzed from the public database GEO. P4HA1 mRNA was found to be significantly upregulated in CRC tissues (GEO/GDS4382 and GEO/GDS5232; $\mathrm{P}<0.001)$ compared with normal tissues (Fig. 1A and B). Overexpression of P4HA1 was also confirmed in CRC clinical samples compared with adjacent normal colorectal tissues from 30 patients with CRC by RT-qPCR (Fig. 1C). In addition, immunohistochemical staining analysis for P4HA1 in CRC tissues found that P4HA1 staining was stronger in CRC tissues compared with adjacent normal tissues (Fig. 1D). P4HA1 protein expression levels were also analyzed in three CRC cell lines (SW480, SW620 and HCT116 cells) via western blotting (Fig. 1E). The results showed a significant difference in the expression levels of P4HA1 in SW620 and HCT116 cells compared with the SW480 cell line, therefore all further in vitro studies were undertaken using these two cell lines.

P4HAl knockdown inhibits CRC cell proliferation. To investigate whether P4HA1 affects the proliferation of SW620 and HCT116 cells, P4HA1 was knocked down using siRNA, which was confirmed via western blotting (Fig. 2A). The P4HA1-knocked down SW620 and HCT116 cells were then subjected to CCK- 8 and MTS cell proliferation assays. The results indicated that P4HA1 knockdown significantly decreased SW620 and HCT116 cell proliferation (Fig. 2B and C). Cell cycle analysis further confirmed the inhibitory effect of P4HA1 knockdown on cell proliferation and more cells were arrested in phase $G_{0} / G_{1}$ (Fig. 2D).

P4HA1 knockdown decreases CRC cell stemness. In order to determine the role of P4HA1 in CRC cell stemness, SW620 and 
A

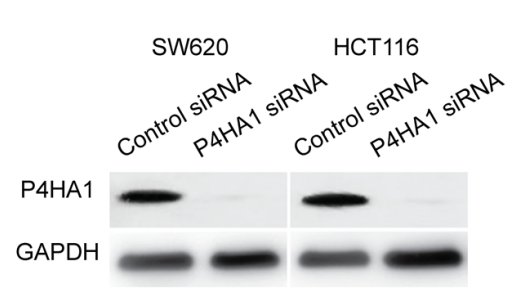

C

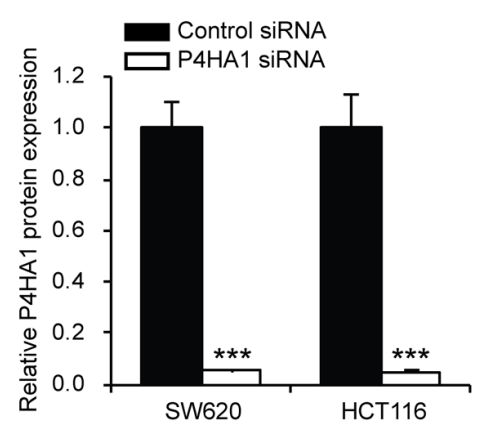

B

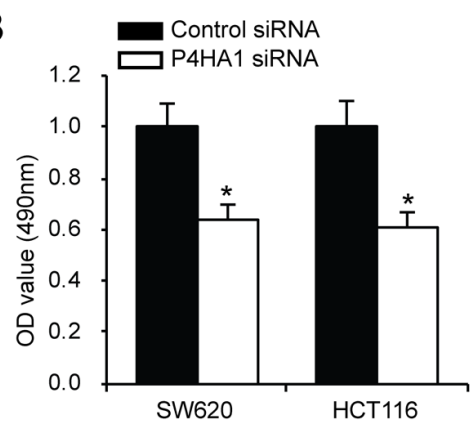

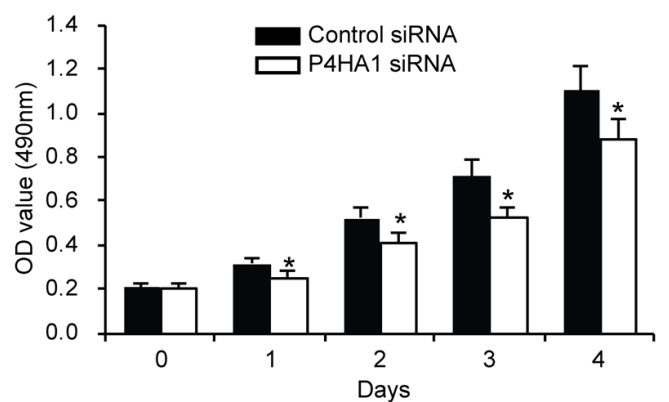

D

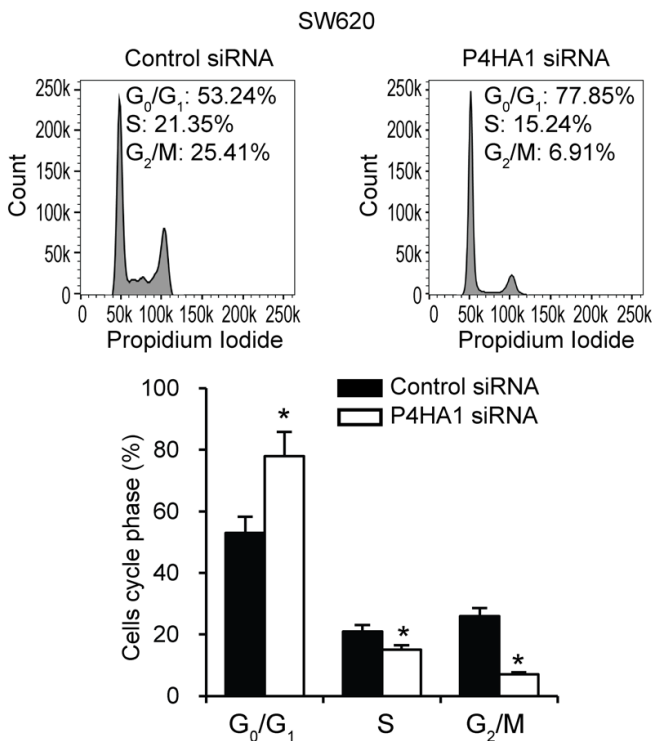

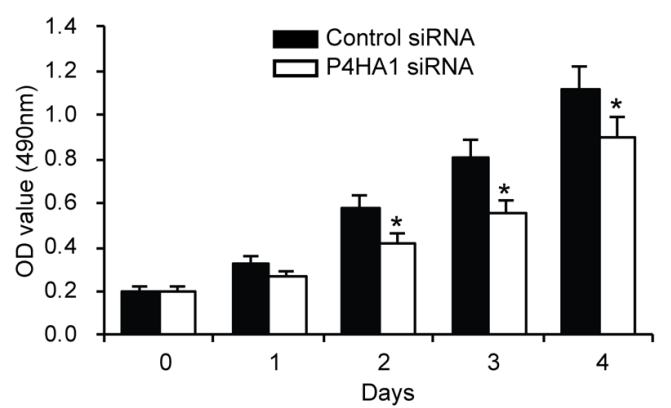

HCT116

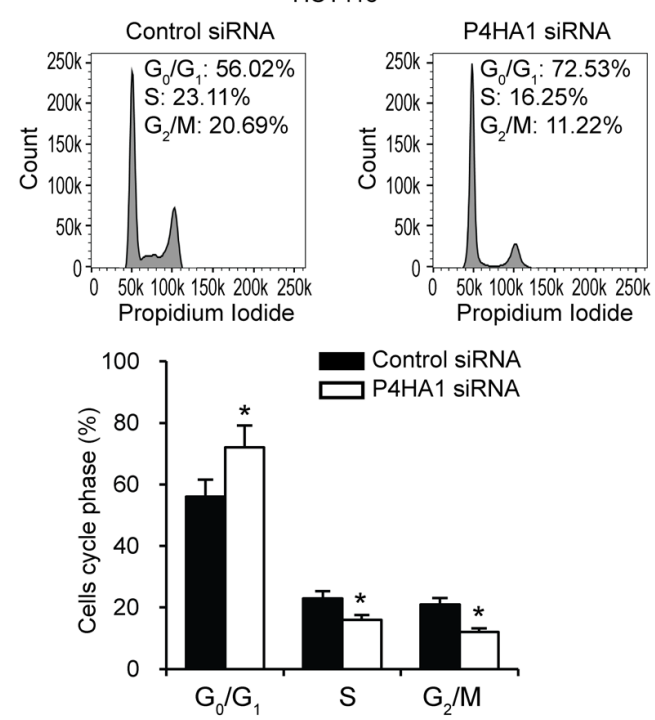

Figure 2. P4HA1 knockdown inhibits colorectal cancer cell proliferation. (A) Western blot analysis of P4HA1 expression in SW620 and HCT116 cells transfected with P4HA1 siRNA or control siRNA. P4HA1 protein expression was quantified in the right panel. (B) Cell proliferation was examined by Cell Counting Kit-8 assays in SW620 and HCT116 cells after P4HA1 knockdown. (C) MTS cell proliferation assay in SW620 and HCT116 cells after P4HA1 knockdown at different time points (0,1,2,3 and 4 days). (D) Cell cycle was examined in SW620 and HCT116 cells upon P4HA1 knockdown. The cells in all phases were analyzed. ${ }^{\mathrm{P}}<0.05,{ }^{* * *} \mathrm{P}<0.001$ vs. Control siRNA. P4HA1, prolyl 4-hydroxylase subunit $\alpha 1$; siRNA, small interfering RNA.

HCT116 cells were cultured in stem cell medium, following examination of the stem cell markers CD133 and Nanog. Knockdown of P4HA1 was found to significantly decrease the expression levels of these stem cell markers (Fig. 3A). Tumorsphere formation assay for SW620 and HCT116 cells showed that P4HA1 knockdown resulted in a decreased formation of spheres (Fig. 3B). Furthermore, the isolation of single CRC cells from primary spheres and the culture of these in stem cell medium, demonstrated that the tumorsphere volume was significantly decreased in P4HA1 knocked down cells (Fig. 3C).
P4HAl knockdown decreases CRC cell chemoresistance. To examine whether P4HA1 knockdown affects SW620 and HCT116 cells chemoresistance, cells were treated with the chemotherapeutic agent 5-Fluorouracil (5-FU). Western blotting analysis showed induced expression of P4HA1 after 5-FU treatment (Fig. 4A). We tested caspase-3 activity to analyze whether P4HA1 silencing affected CRC apoptosis following 5-FU treatment. Increased caspase-3 activity was observed following P4HA1 knockdown (Fig. 4B). The cell viability was analyzed by MTS assays in SW620 and HCT116 cells upon P4HA1 knockdown. The 

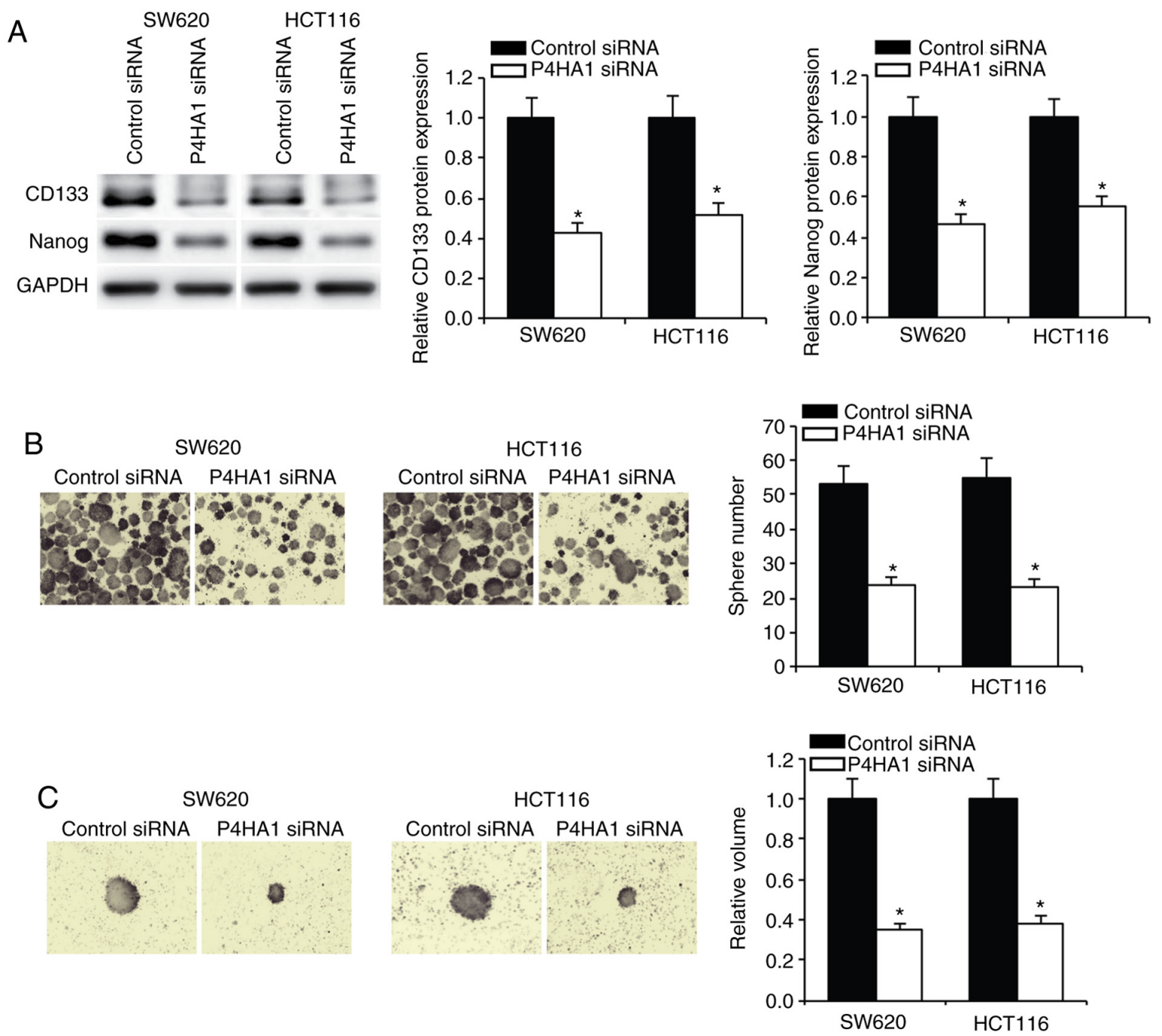

Figure 3. P4HA1 knockdown decreases colorectal cancer cell stemness. (A) CD133 and Nanog expression in SW620 and HCT116 cells with P4HA1 knockdown cultured in stem cell growing medium. Protein expression was quantified in the right panel. (B) Sphere number of P4HA1 knockdown SW620 and HCT116 cells cultured in stem cell growing medium. (C) Relative tumorsphere volume of single SW620 and HCT116 cells with P4HA1 knockdown cultured in stem cell growing medium after 14 days. ${ }^{\mathrm{P}}<0.05$ vs. Control siRNA. P4HA1, prolyl 4-hydroxylase subunit $\alpha 1$.

results showed that knockdown of P4HA1 in the SW620 and HCT116 cells decreased their viability (Fig. 4C). Notably, when comparing the P4HA1 protein expression levels in P4HA1 knocked down cells with or without 5-FU treatment, no obvious change in $\mathrm{P} 4 \mathrm{HA} 1$ expression was observed (Fig. S1). 5-FU failed to affect P4HA1 expression in P4HA1 knockdown cells. The mechanism of the effect of 5-FU treatment in CRC cells with P4HA1 knockdown is worth investigation in the future.

P4HAl promotes CRC through HIFI $\alpha$. To further investigate the mechanism of P4HA1 in the regulation of CRC cell proliferation, stemness and chemoresistance, the expression of HIF1 $\alpha$ was analyzed in the present study. HIF1 $\alpha$ plays a pivotal role in CRC malignancy (20). SW620 cells were cultured under hypoxic conditions of $1 \%$ oxygen atmosphere, and P4HA1 and HIF1 $\alpha$ expression levels were examined after $24 \mathrm{~h}$. The results showed that P4HA1 and HIF1 $\alpha$ could be induced under a hypoxic environment (Fig. 5A). Furthermore, it was revealed that knockdown of P4HA1 resulted in a decreased level of HIF1a, whereas the knockdown of HIF1a did not affect the expression level of p4HA1 under hypoxic conditions (Fig. 5A). We decreased the expression of HIF1 $\alpha$ by siRNA method and performed MTS, tumorsphere formation and caspase-3 activity assays. Knockdown of HIF1 $\alpha$ significantly inhibited CRC cell malignancy (Fig. 5B-D).

P4HAl affects CRC cell Wnt signaling. HIF1 $\alpha$ is associated with Wnt signaling to regulate cancer cell tumorigenesis (21). HIF1 $\alpha$ was found to be a protein that is downstream of P4HA1. In order to investigate whether P4HA1 affects Wnt signaling, P4HA1 was knocked down in SW620 and HCT116 cells, and $\beta$-catenin and $\mathrm{p}-\beta$-catenin protein expression levels were examined via western blotting. The results revealed that knockdown of P4HA1 significantly decreased $\beta$-catenin and increased p- $\beta$-catenin expression (Fig. 6). Subsequently, the examination of the protein expression levels of gene targets of the Wnt signaling pathway (Axin2 and c-Myc) showed that P4HA1 knockdown significantly decreased Axin2 and c-Myc expression in SW620 and HCT116 cells (Fig. 6). This finding indicated the important role of P4HA1/HIF1 $/$ /Wnt signaling axis in CRC. 
A

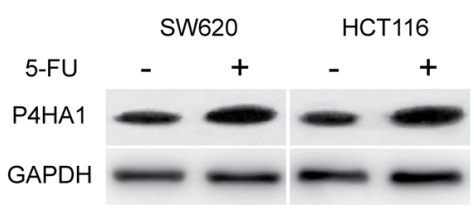

C

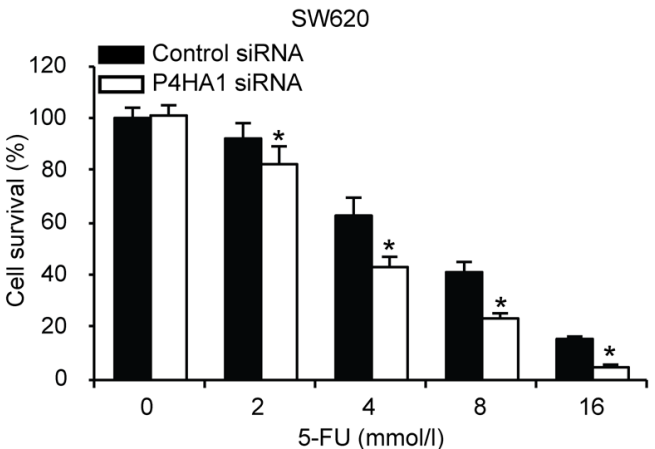

B

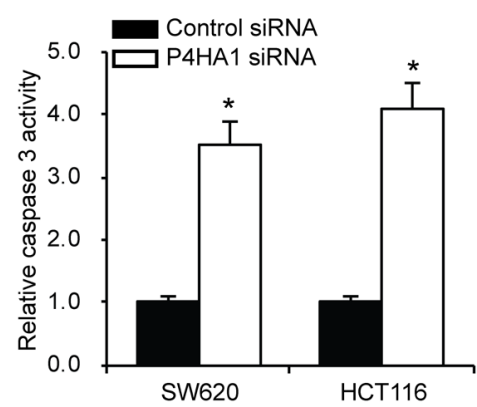

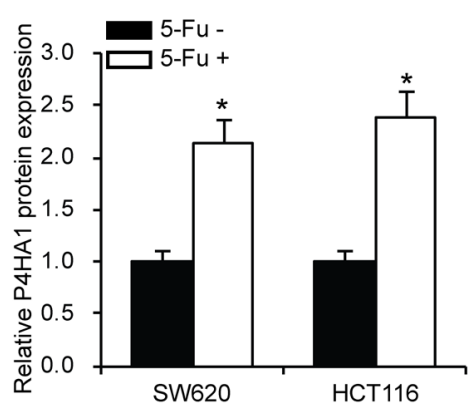

HCT116

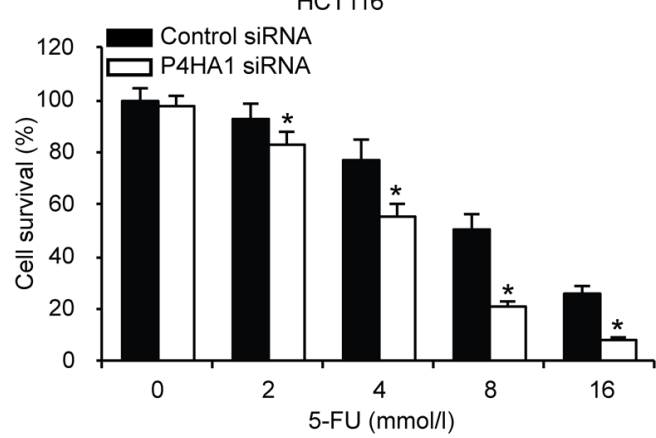

Figure 4. P4HA1 knockdown decreases colorectal cancer cell chemoresistance. (A) Western blot analysis of P4HA1 protein expression in SW620 and HCT116 cells treated with 5-FU. Protein expression was quantified in the right panel. "P<0.05 vs. 5-FU. (B) Relative caspase-3 activity was examined in SW620 and HCT116 cells treated with 5-FU. "P<0.05 vs. Control siRNA. (C) Cell viability in SW620 and HCT116 cells after P4HA1 knockdown treated with different concentrations of 5-FU (0, 2, 4, 8 and $16 \mathrm{mmol} / \mathrm{l})$. $\mathrm{P}<0.05$ vs. Control siRNA. P4HA1, prolyl 4-hydroxylase subunit $\alpha 1$; 5-FU, 5-fluorouracil; siRNA, small interfering RNA.

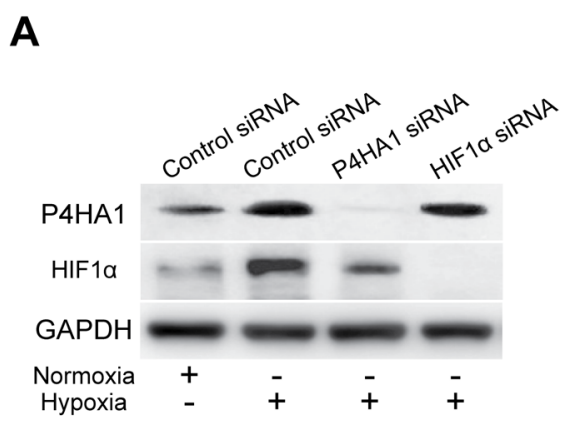

B

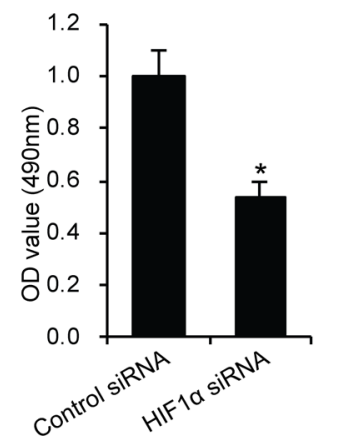

C
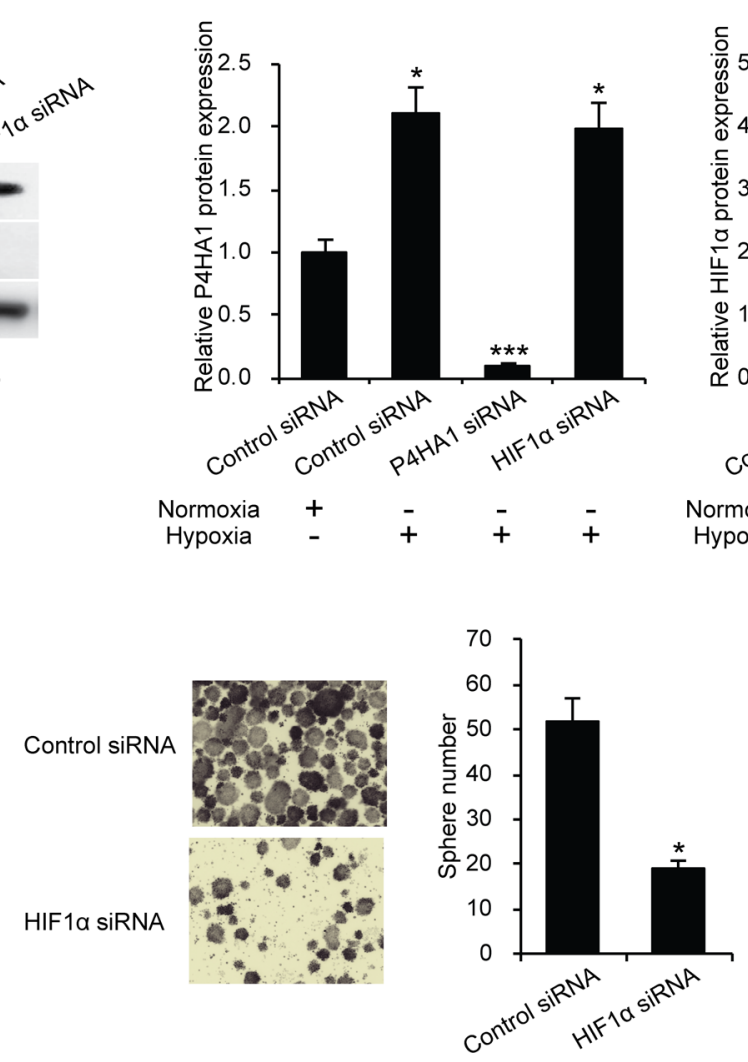

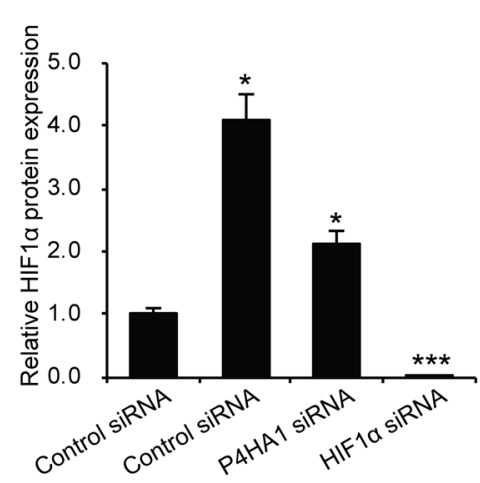

D

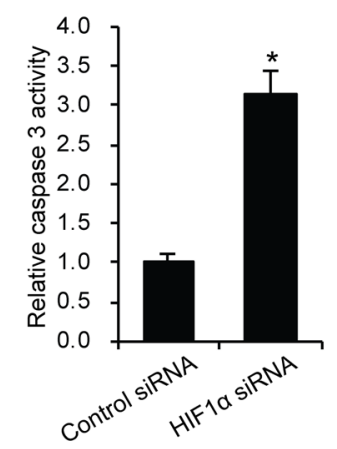

Figure 5. P4HA1 regulates proliferation, stemness and apoptosis of colorectal cancer cells through HIF1 $\alpha$. (A) P4HA1 and HIF1 $\alpha$ expression was examined in SW620 cells under hypoxia. Protein expression was quantified in the right panel. ${ }^{*} \mathrm{P}<0.05,{ }^{* * * *} \mathrm{P}<0.001$ vs. control siRNA without hypoxia. Examination of (B) cell proliferation, (C) sphere number and (D) caspase-3 activity in SW620 cells upon HIF1 $\alpha$ knockdown. "P<0.05. P4HA1, prolyl 4-hydroxylase subunit $\alpha 1$; HIF1 $\alpha$, hypoxia inducible factor-1 $\alpha$; siRNA, small interfering RNA. 

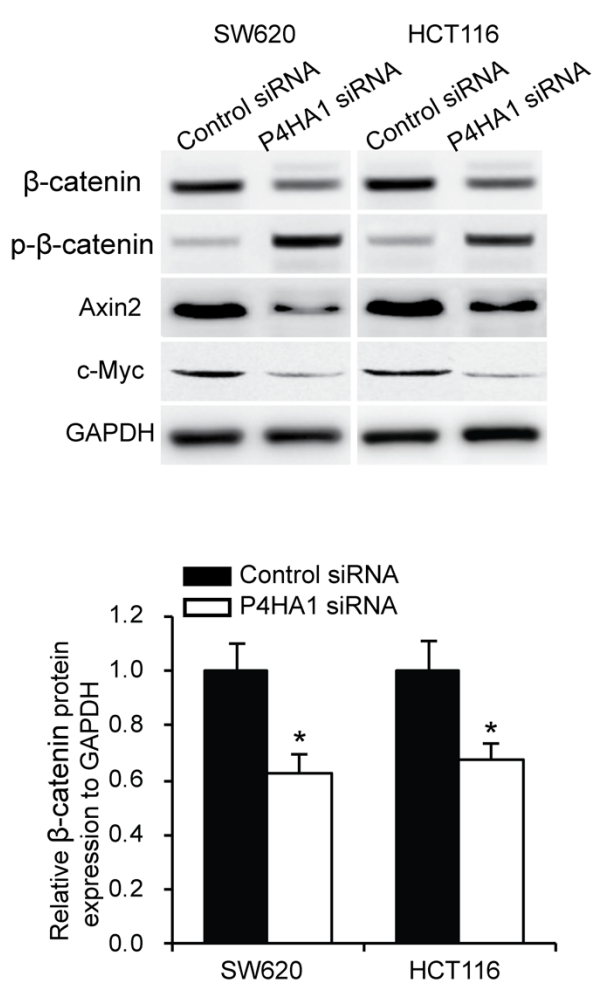
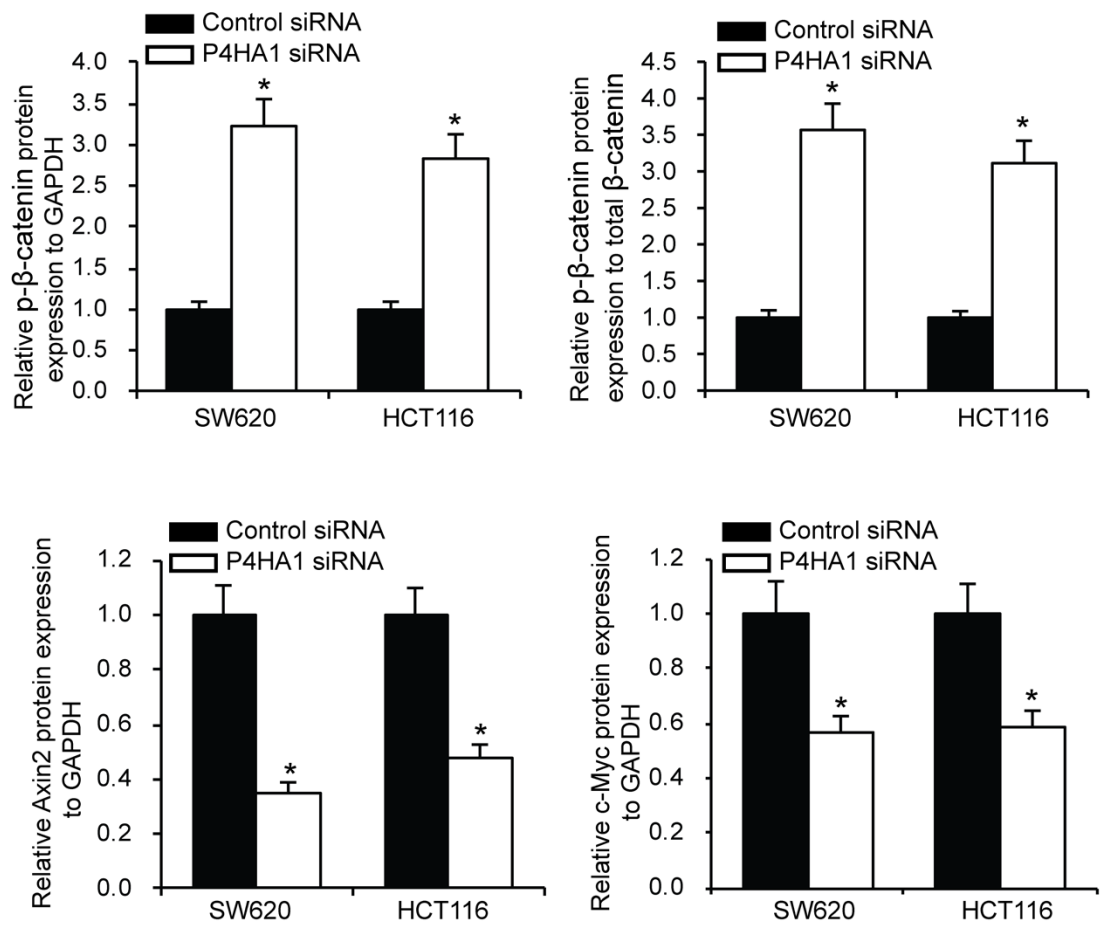

Figure 6. P4HA1 affects CRC cell Wnt signaling. Western blot analysis of $\beta$-catenin, p- $\beta$-catenin, Axin 2 and c-Myc in SW620 and HCT116 cells after P4HA1 knockdown. Protein expression levels was quantified in the right panel. * $\mathrm{P}<0.05$ vs. Control siRNA. P4HA1, prolyl 4-hydroxylase subunit $\alpha 1$; p-, phosphorylated; Axin2, axis inhibition protein 2; Wnt, wingless-type mouse mammary tumor virus integration site family; siRNA, small interfering.

\section{Discussion}

The present study is a preliminary demonstration of the assessment of a PTM in the carcinogenesis of CRC. The results from the current study strengthen the genomic and epigenomic findings in CRC biomarker discovery, suggesting that the study of PTMs, especially P4HA1, could have an important prognostic value and work as potential therapeutic targets.

Through a review of GEO datasets (22) the expression of P4HA1 was found to be significantly upregulated in CRC $(16,17,23)$. This is also seen in in vitro assays in human CRC tissue samples. These observations are in concordance with the findings in triple negative human breast cancer $(10,11)$, adenopancreatic carcinoma (9), gliomas (24) and oral squamous cell carcinoma (25). P4HA1 has been reported to be regulated by miR-124, which is a potential target in CRC treatment (26). Further in vitro assays using the CRC cell lines SW480, SW620 and HCT116 showed that P4HA1 is expressed in these cells. However, the expression of P4HA1 was found to be lower in SW480 cells compared with the other two cell lines. It has previously been shown that the expression of proteins can significantly differ amongst primary CRC cells (SW480) vs. metastatic colon cancer cell lines such as (SW620 and HCT116) (27).

As aforementioned, P4HA1 is the most abundantly found isoform of prolyl 4-hydroxylase and is responsible for 4-hydroxylation of proline residues on collagen (11). This post-translational modification is responsible for the appropriate folding and tertiary structure of collagen (10). Collagen is the most abundant protein found in the extracellular matrix
(ECM); the ECM being the acellular component within tissues and organs (28). The role of ECM extends beyond providing merely a scaffold and/or a physical support to cells. It also provides the appropriate milieu for biochemical cues essential for the maintenance of tissue homeostasis (28).

Tumor-derived ECM is not only stiffer, but it is also biochemically distinct than the normal ECM. Besides supporting cancer cells, the role of ECM in cellular functions such as cell proliferation, cell migration, differentiation and survival have been well described $(29,30)$. The role of collagen in mediating these cellular features has been previously reported (31). It is likely that overexpression of collagen may promote epithelial-mesenchymal transition, tumor initiating potential and self-renewal of CSCs, thereby mediating the stemness phenotype $(29,32)$.

These findings may in part explain the present results, wherein silencing of P4HA1 downregulates cellular proliferation, reduced cancer stemness and decreased chemoresistance plausibly through the breakdown of the ECM. As has been demonstrated previously, it is likely that the silencing of P4HA1 decreases the turnover of collagen in the matrix (33). The absence of collagen abrogates activation of the focal adhesion kinase (FAK) through $\beta 1$-integrin (34). This in turn would downregulate multiple FAK effector molecules such as the phosphatidyl inositol 3 kinase or growth factor receptor bound protein 7 and cell division control protein 42 , thereby regulating various cell processes, such as division and migration.

Studies in breast cancer have also found a link between collagen hydroxylation and HIF1 $\alpha$ activation during tumor 
progression (10). Increased expression of P4HA1 was found to enhance stability of HIF1 $\alpha$ by significantly reducing the levels of cellular $\alpha$-ketoglutarate and increasing the levels of succinate (11). Similar observations were also observed in the present study, with the expression of P4HA1 being demonstrated to be associated with increased stability of HIF1 $\alpha$. Furthermore, HIF1 $\alpha$ is associated with a poor prognosis in CRC (35). HIF1 $\alpha$ plays an angiogenic role in CRC through the upregulation of angiogenic factors such as VEGF (36).

The present findings also suggested inactivation of the Wnt signaling pathway by silencing of P4HA1. There are two possible explanations for the observations: i) It is likely that P4HA1 negatively regulates the multiprotein dissociation complex of the Wnt pathway, thereby reducing phosphorylation and consequent proteasomal degradation of $\beta$-catenin. This in part would be explained by the downregulation of Axin 2 upon silencing of P4HA1; and ii) hypoxia and HIF1 $\alpha$ regulates the expression of several transcriptional activators, such as B cell lymphoma 9 (BCL9), which in turn regulates a number of genes in the Wnt pathway, namely CD44, Axin2 and survivin. It has been shown that BCL9 knockouts have significantly decreased expression of these genes (37).

In conclusion, as the majority of CRC cases present with an aberrated canonical Wnt signaling pathway, it would be essential to target molecules that deregulate the Wnt pathway. The results of the present study indicate that investigation into P4HA1 as a target biomolecule to predict prognosis of CRC could be beneficial. Alongside this, therapeutic targets to selectively downregulate or silence the expression of P4HA1 may be investigated to target P4HA1-mediated CRC pathogenesis.

\section{Acknowledgements}

Not applicable.

\section{Funding}

The present study was funded by the General program of Qiqihar Academy of Medical Sciences (grant no. QMSI2019M-26).

\section{Availability of data and materials}

The datasets used and/or analyzed during the current study are available from the corresponding author on reasonable request. The data from GEO databases were cited in the article.

\section{Authors' contributions}

ZY designed the study. QZ, YY, HZ, YS, WZ and ZY performed the experiments. QZ, YY, TL and YH defined the analysis strategy and performed the statistical analysis. QZ wrote the first draft of the article. All authors read and approved the final version.

\section{Ethics approval and consent to participate}

The present study was performed in accordance with the Declaration of Helsinki and approved by the Medical Ethics Committee of Qiqihar Medical University (no. 2015QY137).
All patients provided written informed consent prior to the study start.

\section{Patient consent for publication}

Not applicable.

\section{Competing interests}

The authors declared that they have no competing interests.

\section{References}

1. Keum N and Giovannucci E: Global burden of colorectal cancer: Emerging trends, risk factors and prevention strategies. Nat Rev Gastroenterol Hepatol 16: 713-732, 2019.

2. Dekker E, Tanis PJ, Vleugels JLA, Kasi PM and Wallace MB: Colorectal cancer. Lancet 394: 1467-1480, 2019.

3. Bray F, Ferlay J, Soerjomataram I, Siegel RL, Torre LA and Jemal A: Global cancer statistics 2018: GLOBOCAN estimates of incidence and mortality worldwide for 36 cancers in 185 countries. CA Cancer J Clin 68: 394-424, 2018.

4. Siegel RL, Miller KD and Jemal A: Cancer statistics, 2019. CA Cancer J Clin 69: 7-34, 2019.

5. Kuipers EJ, Grady WM, Lieberman D, Seufferlein T, Sung JJ, Boelens PG, van de Velde CJ and Watanabe T: Colorectal cancer. Nat Rev Dis Primers 1: 15065, 2015.

6. Martini G, Troiani T, Cardone C, Vitiello P, Sforza V, Ciardiello D, Napolitano S, Della Corte CM, Morgillo F, Raucci A, et al: Present and future of metastatic colorectal cancer treatment: A review of new candidate targets. World J Gastroenterol 23: 4675-4688, 2017

7. Locke WJ, Guanzon D, Ma C, Liew YJ, Duesing KR, Fung KYC and Ross JP: DNA methylation cancer biomarkers: Translation to the clinic. Front Genet 10: 1150, 2019.

8. Chakraborty S, Hosen MI, Ahmed M and Shekhar HU: Onco-multi-OMICS approach: A new frontier in cancer research. Biomed Res Int 2018: 9836256, 2018.

9. Cao XP, Cao Y,Li WJ,Zhang HH and Zhu ZM: P4HA1/HIF1alpha feedback loop drives the glycolytic and malignant phenotypes of pancreatic cancer. Biochem Biophys Res Commun 516: 606-612, 2019.

10. Xu R: P4HA1 is a new regulator of the HIF-1 pathway in breast cancer. Cell Stress 3: 27-28, 2019.

11. Xiong G, Stewart RL, Chen J, Gao T, Scott TL, Samayoa LM, O'Connor K, Lane AN and Xu R: Collagen prolyl 4-hydroxylase 1 is essential for HIF-1alpha stabilization and TNBC chemoresistance. Nat Commun 9: 4456, 2018.

12. Chakravarthi BV, Pathi SS, Goswami MT, Cieślik M, Zheng H, Nallasivam S, Arekapudi SR, Jing X, Siddiqui J, Athanikar J, et al: The miR-124-prolyl hydroxylase P4HA1-MMP1 axis plays a critical role in prostate cancer progression. Oncotarget 5: 6654-6669, 2014.

13. Schatoff EM, Leach BI and Dow LE: Wnt signaling and colorectal cancer. Curr Colorectal Cancer Rep 13: 101-110, 2017.

14. Zhan T, Rindtorff $\mathrm{N}$ and Boutros $\mathrm{M}$ : Wnt signaling in cancer. Oncogene 36: 1461-1473, 2017.

15. Yoshida N, Kinugasa T, Ohshima K, Yuge K, Ohchi T, Fujino S, Shiraiwa S, Katagiri M and Akagi Y: Analysis of Wnt and beta-catenin expression in advanced colorectal cancer. Anticancer Res 35: 4403-4410, 2015.

16. Khamas A, Ishikawa T, Shimokawa K, Mogushi K, Iida S, Ishiguro M, Mizushima $\mathrm{H}$, Tanaka $\mathrm{H}$, Uetake $\mathrm{H}$ and Sugihara $\mathrm{K}$ : Screening for epigenetically masked genes in colorectal cancer Using 5-Aza-2'-deoxycytidine, microarray and gene expression profile. Cancer Genomics Proteomics 9: 67-75, 2012.

17. Danielsen SA, Cekaite L, Ågesen TH, Sveen A, Nesbakken A, Thiis-Evensen E, Skotheim RI, Lind GE and Lothe RA: Phospholipase $\mathrm{C}$ isozymes are deregulated in colorectal cancer-insights gained from gene set enrichment analysis of the transcriptome. PLoS One 6: e24419, 2011.

18. Flejou JF: WHO Classification of digestive tumors: The fourth edition. Ann Pathol 31 (Suppl 5): S27-S31, 2011 (In French).

19. Livak KJ and Schmittgen TD: Analysis of relative gene expression data using real-time quantitative PCR and the 2(-Delta Delta C(T)) method. Methods 25: 402-408, 2001. 
20. Nagaraju GP, Bramhachari PV, Raghu G and El-Rayes BF Hypoxia inducible factor-1alpha: Its role in colorectal carcinogenesis and metastasis. Cancer Lett 366: 11-18, 2015.

21. Giles RH, Lolkema MP, Snijckers CM, Belderbos M, van der Groep P, Mans DA, van Beest M, van Noort M, Goldschmeding R, van Diest PJ, et al: Interplay between VHL/HIFlalpha and Wnt/beta-catenin pathways during colorectal tumorigenesis. Oncogene 25: 3065-3070, 2006.

22. Barrett T, Wilhite SE, Ledoux P, Evangelista C, Kim IF Tomashevsky M, Marshall KA, Phillippy KH, Sherman PM, Holko M, et al: NCBI GEO: Archive for functional genomics data sets-update. Nucleic Acids Res 41 (Database issue): D991-D995, 2013.

23. Ågesen TH, Berg M, Clancy T, Thiis-Evensen E, Cekaite L, Lind GE, Nesland JM, Bakka A, Mala T, Hauss HJ, et al: CLC and IFNAR1 are differentially expressed and a global immunity score is distinct between early- and late-onset colorectal cancer. Genes Immun 12: 653-662, 2011.

24. Hu WM, Zhang J, Sun SX, Xi SY, Chen ZJ, Jiang XB, Lin FH, Chen ZH, Chen YS, Wang J, et al: Identification of P4HA1 as a prognostic biomarker for high-grade gliomas. Pathol Res Pract 213: 1365-1369, 2017.

25. Kappler M, Kotrba J, Kaune T, Bache M, Rot S, Bethmann D, Wichmann H, Güttler A, Bilkenroth U, Horter S, et al: P4HA1: A single-gene surrogate of hypoxia signatures in oral squamous cell carcinoma patients. Clin Transl Radiat Oncol 5: 6-11, 2017

26. Agarwal S, Behring M, Kim HG, Bajpai P, Chakravarthi BVSK, Gupta N, Elkholy A, Al Diffalha S, Varambally S and Manne U: Targeting P4HA1 with a small molecule inhibitor in a colorectal cancer PDX model. Transl Oncol 13: 100754, 2020.

27. Xiang L, Mou J, Shao B, Wei Y, Liang H, Takano N, Semenza GL and Xie G: Glutaminase 1 expression in colorectal cancer cells is induced by hypoxia and required for tumor growth, invasion, and metastatic colonization. Cell Death Dis 10: 40, 2019.

28. Frantz C, Stewart KM and Weaver VM: The extracellular matrix at a glance. J Cell Sci 123: 4195-4200, 2010.

29. Begum A, Ewachiw T, Jung C, Huang A, Norberg KJ, Marchionni L, McMillan R, Penchev V, Rajeshkumar NV, Maitra A, et al: The extracellular matrix and focal adhesion kinase signaling regulate cancer stem cell function in pancreatic ductal adenocarcinoma. PLoS One 12: e0180181, 2017.
30. Poltavets V, Kochetkova M, Pitson SM and Samuel MS: The role of the extracellular matrix and its molecular and cellular regulators in cancer cell plasticity. Front Oncol 8: 431, 2018.

31. Somaiah C, Kumar A, Mawrie D, Sharma A, Patil SD, Bhattacharyya J, Swaminathan R and Jaganathan BG: Collagen promotes higher adhesion, survival and proliferation of mesenchymal stem cells. PLoS One 10: e0145068, 2015.

32. Nallanthighal S, Heiserman JP and Cheon DJ: The role of the extracellular matrix in cancer stemness. Front Cell Dev Biol 7: 86, 2019.

33. Zou Y, Donkervoort S, Salo AM, Foley AR, Barnes AM, Hu Y, Makareeva E, Leach ME, Mohassel P, Dastgir J, et al: P4HA1 mutations cause a unique congenital disorder of connective tissue involving tendon, bone, muscle and the eye. Hum Mol Genet 26: 2207-2217, 2017.

34. Zhao X and Guan JL: Focal adhesion kinase and its signaling pathways in cell migration and angiogenesis. Adv Drug Deliv Rev 63: 610-615, 2011.

35. Baba Y, Nosho K, Shima K, Irahara N, Chan AT, Meyerhardt JA, Chung DC, Giovannucci EL, Fuchs CS and Ogino S: HIF1A overexpression is associated with poor prognosis in a cohort of 731 colorectal cancers. Am J Pathol 176: 2292-2301, 2010

36. Huang ZY, Zhang LH, Zhao C, Liu R, Tong H, Gan C, Lan T, Tang CW and Gao JH: High HIF-1 $\alpha$ expression predicts poor prognosis of patients with colon adenocarcinoma. Int J Clin Exp Pathol 11: 5635-5646, 2018.

37. Xu W, Zhou W, Cheng M, Wang J, Liu Z, He S, Luo X, Huang W, Chen T, Yan W and Xiao J: Hypoxia activates Wnt/ $\beta$-catenin signaling by regulating the expression of BCL9 in human hepatocellular carcinoma. Sci Rep 7: 40446, 2017.

This work is licensed under a Creative Commons Attribution-NonCommercial-NoDerivatives 4.0 International (CC BY-NC-ND 4.0) License. 